\title{
YACHAY QUECHUA: PLATAFORMA WEB PARA PROMOVER EL APRENDIZAJE DEL QUECHUA CUSQUEÑO MEDIANTE TECNOLOGÍAS DE LA INFORMACIÓN
}

\section{Yachay quechua: web platform to promote the learning of quechua from Cusco using information technologies}

\author{
Luigi Salas Miranda ${ }^{1,}$ \\ 1 Universidad Andina del Cusco, Cusco, Perú. \\ ”luigism18@gmail.com
}

\begin{abstract}
Resumen
El Objetivos de la investigación fue promover y revalorar nuestra lengua materna -el quechua cusqueño- mediante tecnologías de información de acuerdo a diferentes estilos de aprendizaje. Entre los materiales y métodos para la obtención de datos se consideró el análisis de libros, revistas y artículos. Estas fuentes se consiguieron de bases de datos bibliográficas como: ScienceDirect, Scopus, Scielo y Alicia. Del mismo modo, se realizaron encuestas a ciudadanos cusqueños con el fin de saber los motivos causantes de la desidia por aprender el quechua y qué aspectos quisieran que fueran mejorados en la enseñanza actual del quechua. Se tuvo como resultado la monotonía de alternativas en el aprendizaje del quechua cusqueño, así como la poca valorización que se le da, las cuales son las principales razones por lo que el porcentaje de quechua-hablantes disminuye considerablemente cada año, es por eso que nos enfocamos en estos pilares para contrarrestar esta problemática social y cultural, mediante tecnologías de vanguardia y que están siendo usadas en otros países con fines y propósitos educativos de calidad. Entre las conclusiones se mencionan: el uso de tecnologías de información para el uso y el aprendizaje del quechua cusqueño, adecuados a los estilos de aprendizaje contrarrestará la disminución de quechua-hablantes en el país, puesto que, al tener más variedad y elegir la herramienta para su aprendizaje, aumentará en los jóvenes el interés por aprenderlo como lo hacen actualmente aprendiendo otras materias e incluso, otras lenguas extranjeras.

Palabras clave: Plataforma web, tecnologías de información, quechua cusqueño, aplicaciones móviles, E-learning (Fuente: ScienceDirect, Scoppus, Scielo, Alicia).
\end{abstract}

\begin{abstract}
The objectives of the research were to promote and revalue our mother tongue-Quechua-Cusco-through information technologies according to different learning styles. Among the materials and methods for obtaining data, the analysis of books, magazines and articles was considered. These sources were obtained from bibliographic databases such as: ScienceDirect, Scopus, Scielo and Alicia. In the same way, surveys were carried out to citizens of Cusco with the purpose of knowing the reasons that caused the apathy to learn Quechua and what aspects they wanted to improve in the current teaching of Quechua. The result was the monotony of alternatives in the learning of Quechua from Cusco, as well as the low value given to it, which are the main reasons why the percentage of Quechua speakers decreases considerably every year, that is why we We focus on these pillars to counteract this social and cultural problem, using cutting-edge technologies and which are being used in other countries for quality educational purposes and purposes. Among the conclusions are mentioned: the use of information technologies for the use and learning of Quechua from Cusco, adapted to the learning styles, will counteract the decrease in Quechua-speakers in the country, since, having more variety and choosing the tool for their learning, it will increase in the young people the interest to learn it as they do it at the moment learning other matters and even, other foreign languages.

Keywords: Web platform, information technologies, quechua cusqueño, mobile applications, E-learning (Source: ScienceDirect, Scoppus, Scielo,
\end{abstract} Alicia).

Citar como: Salas, L. (2018). Yachay quechua: plataforma web para promover el aprendizaje del quechua cusqueño mediante tecnologías de la información. Rev Yachay, 7(1), 367-372.

Recibido: 16-09-2018; Aceptado 07-12-2018 


\section{Introducción}

Esta investigación tiene como principal propósito de estudio revalorar nuestra lengua materna: el quechua cusqueño. En concreto, el proyecto que presentamos es con el objetivo de fomentar, promover, e incentivar el uso de nuestra lengua; ya que uno de los principales motivos es la desidia de las personas por aprender y valorar nuestra lengua. De este modo, todo el planteamiento y desarrollo de esta tesis de investigación se fundamenta en una interrogante fundamental: ¿Cómo fomentar, incentivar y promover el uso del quechua cusqueño de acuerdo con los requerimientos del mundo actual? Para realizar este proyecto se hará un estudio de las formas actuales para aprender el quechua, elegir las posibles metodologías a implementar y, finalmente, escoger la o las más viables.

\section{Materiales y métodos}

Para obtener los datos — que se mostrarán posteriormente-, se analizaron: libros, revistas y artículos; estas fuentes se consiguieron de bases de datos bibliográficas como ScienceDirect, Scopus, Scielo y Alicia. Del mismo modo, también nos basamos en otras fuentes, cuya principal plataforma fue YouTube, brindándonos una gran cantidad de videos con diferentes enfoques, pero con la única finalidad de mostrarnos los avances tecnológicos para la enseñanza de idiomas.

Todas las fuentes tuvieron como requisito usar las siguientes palabras clave:

plataforma web, tecnologías de la información, e-learning, quechua, realidad aumentada.

El tipo de investigación (alcance) es aplicativo, ya que se pudo estudiar e indagar en jóvenes cusqueños las causas del desinterés por aprender el quechua.

El nivel de investigación (diseño) es experimental, porque con esta investigación se logró construir una plataforma web que pueda fomentar y promover el uso del quechua basado en los estilos de aprendizaje por medio de las tecnologías de la información.

En cuanto al método de investigación (contrastación de hipótesis) se recurrió al método sintético, puesto que integramos todos los componentes relacionados a nuestro objetivo de estudio, que es la disminución de quechua-hablantes en el Perú y, de esta manera, estudiamos todos los factores y las causas de este problema social; para poder brindar más alternativas de aprendizaje del quechua.

\section{Resultados}

Luego de comprobar que en otros países utilizan tecnologías como la realidad aumentada mediante dispositivos móviles y enfocados a los diferentes estilos de aprendizaje para diferentes materias — como las matemáticas, lingüísticas, y otras ciencias más-, decidimos implementar todas estas herramientas, metodologías y tecnologías para el aprendizaje del quechua cusqueño haciéndola más variada y entretenida para que los jóvenes puedan interactuar con ellas, y aumente su interés en aprenderlo.

\section{Plataforma web}

Página web - www.YachayQuechua.com

Por medio de nuestra página web podrás ver notas de actualidad y contenido traducido en quechua para fomentar el uso del quechua cusqueño.

Figura 1: Imagen de portada de la página web de Yachay Quechua

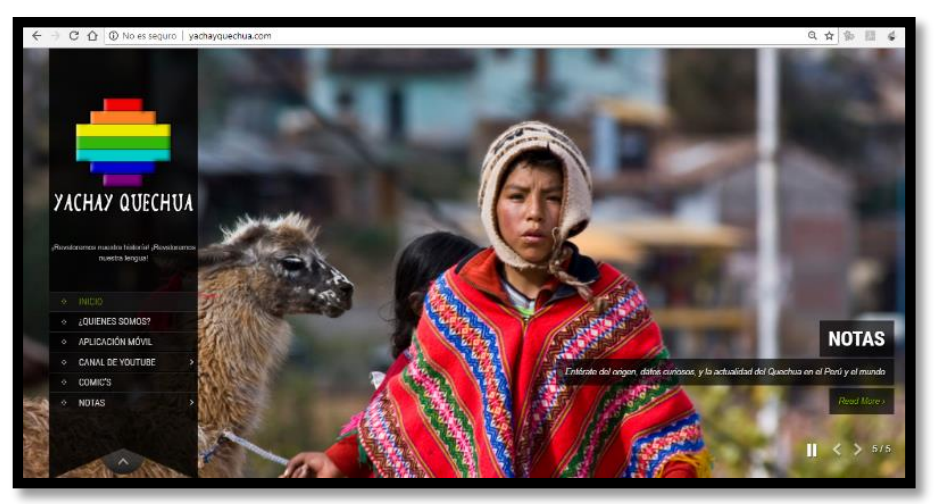

Rev. Yachay volumen (7) Número (1), enero-diciembre 2018 
Aplicación móvil: Disponible solo para Android, próximamente para $i O S$.

En nuestra App también tendrás mucho más contenido y videos del quechua cusqueño en un solo lugar.

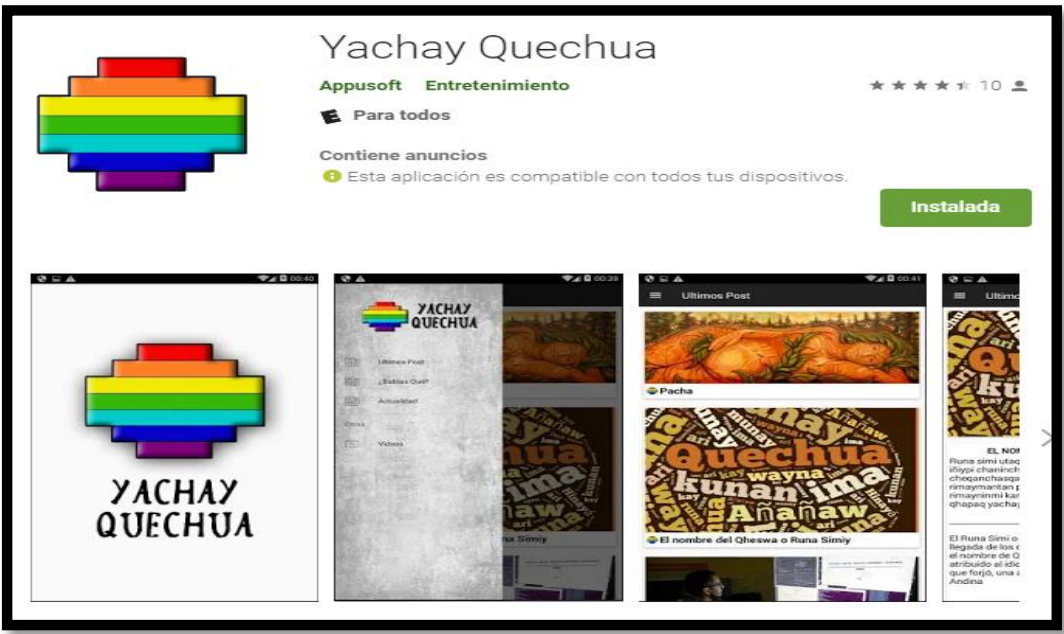

Figura 2: Imagen de la aplicación móvil "Yachay quechua"

Aplicación móvil «Calles del Cusco»

Es una App móvil, la cual da a conocer la historia y el origen del nombre de las calles del Cusco que están en quechua.

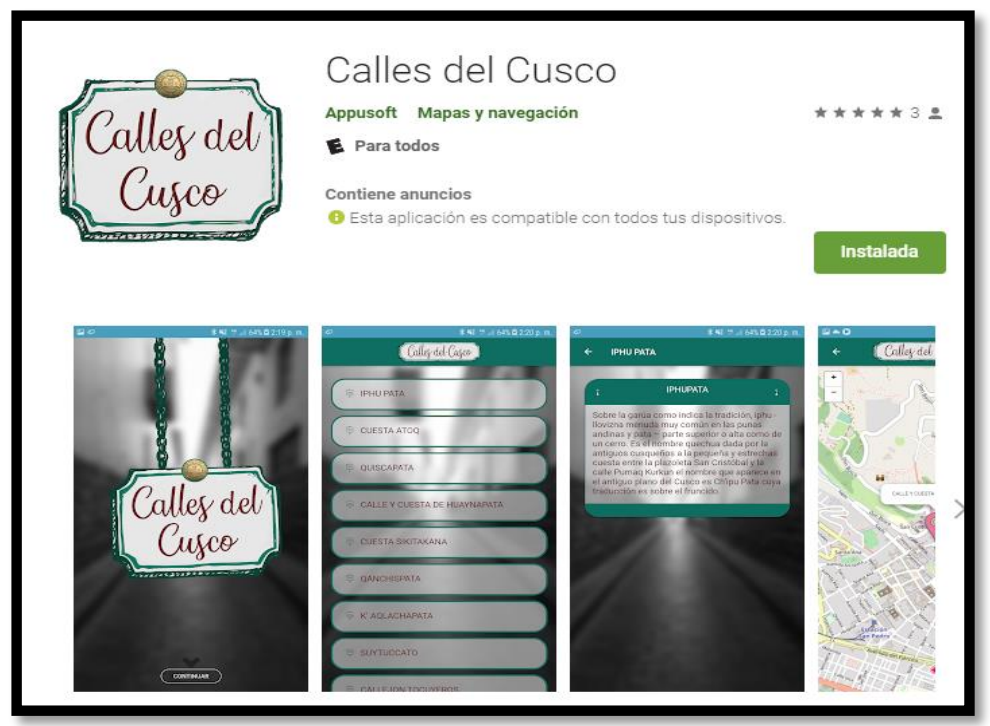

Figura 3: Imagen de la aplicación móvil «Calles del Cusco» 


\section{Videos}

Subimos material audiovisual a nuestro canal de YouTube y a nuestra página de Facebook.

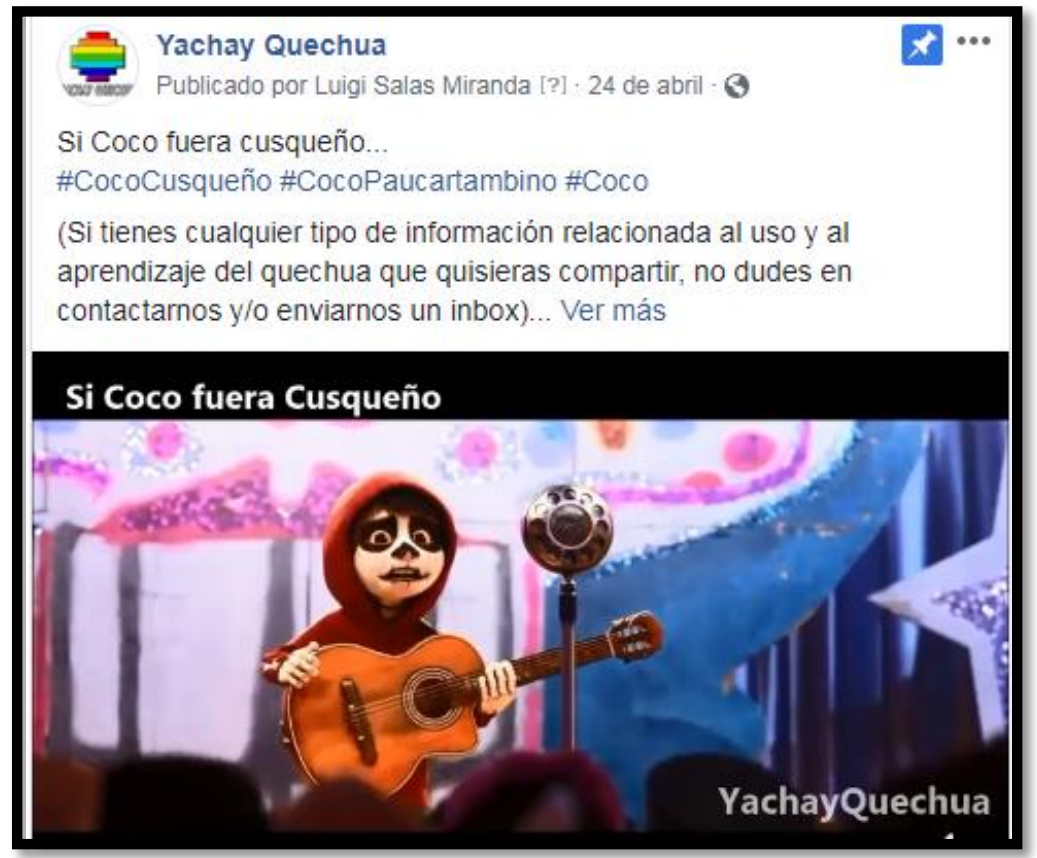

Figura 4: Imagen de la página de Facebook de Yachay Quechua

Comics: Qosqomic con 'realidad aumentada'

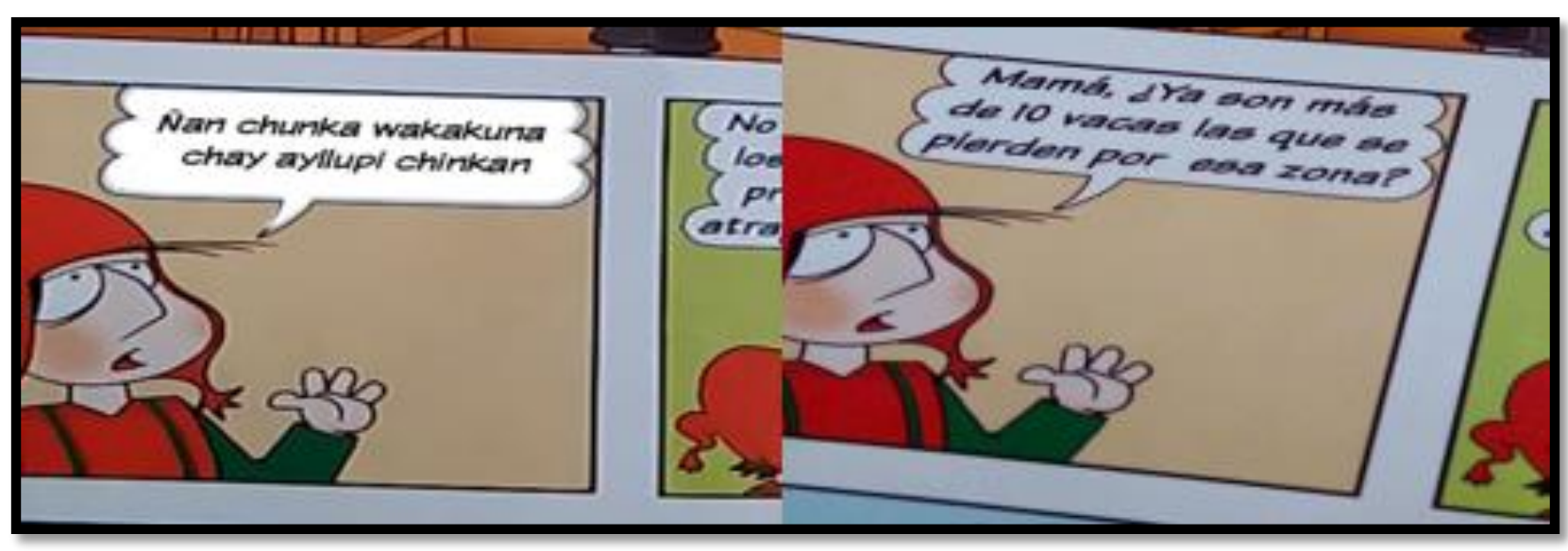

Figura 5: Qosqomic 11 con realidad aumentada y sin realidad aumentada

\section{Discusión}

El propósito de esta investigación no es de competir con la enseñanza de otras lenguas nativas o extranjeras, muy por el contrario: complementarlas ya que aprender el quechua, otra lengua nativa u extranjera; tiene un valor significativo diferente y la importancia de cada una es muy significativa. Por otra parte, el propósito tampoco es competir con entidades que promueven el aprendizaje del quechua como centros de idiomas o academias; Yachay Quechua, simplemente es una alternativa más para fomentar y promover el uso del quechua cusqueño para que los jóvenes puedan revalorarlo y que la cantidad de quechua hablantes no siga disminuyendo considerablemente cada año.

Rev. Yachay volumen (7) Número (1), enero-diciembre 2018 


\section{Conclusiones}

- Al tener el quechua solo dos modalidades de aprendizaje: estudiarlo en un centro de idiomas o aprenderlo por medio de un pariente cercano.

Esto hace que los jóvenes decidan a aprender lenguas extranjeras que aprovechan de mejor manera las tecnologías de información y redes sociales para su aprendizaje, es por ello la demanda de plataformas web que brindan cursos online, y aplicaciones móviles y, por esta razón es que Yachay Quechua aprovecha estas herramientas tecnológicas, además de tecnologías recientes como 'Realidad aumentada' y 'Realidad virtual'.

- No solo nos enfocamos en las herramientas tecnológicas, también en nuestros usuarios, así como sabemos que no todas las personas tienen los mismos estilos de aprendizaje, personas que aprenden idiomas con tan solo tener dos textos en diferentes lenguas; algunos aprenden muchísimo mejor por medios auditivos como la música o grabaciones, otras por medios audiovisuales como videos, filmaciones, realidad aumentada, realidad virtual. Existen otras personas que aprenden por medio de los juegos; lo que se quiere lograr es que, de esta manera, ellos puedan tener alternativas de aprendizaje de acuerdo al gusto de cada usuario de la plataforma.

- Yachay Quechua, por medio de sus tecnologías de información implementadas y, basándose en los estilos de aprendizaje más comunes, promueve alternativas para que los jóvenes, que es nuestro público objetivo de este emprendimiento, pueda fomentarles el uso y aprendizaje de nuestra lengua materna: el quechua cusqueño.

\section{Agradecimientos}

A la Universidad Andina del Cusco, por promover la investigación por medio de eventos, concursos, ferias y publicaciones en sus revistas. Esta investigación fue realizada aproximadamente durante un año, tiempo en el cual la ingeniera Pilar Hidalgo León, fue parte fundamental de la elaboración de este proyecto, ya que sus conocimientos y enseñanzas en aulas universitarias hicieron posible este estudio.

A la hermana Violeta Pilares Vásquez, por todo el apoyo brindado desde el inicio de este emprendimiento, quien me presentó a grandísimas personas como el sr. Gonzalo Valderrama y la sra. Teresa Campos Chong, en la Dirección Desconcentrada de Cultura del Cusco, con los que cuento incondicionalmente para el desarrollo de este emprendimiento social.

Y a mi mamá por la paciencia y el apoyo incondicional en todos mis proyectos.

\section{Contribuciones de autoría}

Yo, Luigi Salas Miranda, soy del CEO y fundador de la StartUp «Yachay Quechua»

\section{Fuentes de financiamiento}

Este proyecto ganó financiamiento producto de sus logros en distintos concursos llevados en la Universidad Andina del Cusco, como Patenta Universidad Andina, Feria de Ciencia y Tecnología, así como también la Dirección Desconcentrada de Cultura del Cusco; por otra parte, nos hubiera encantado recibir el financiamiento del Ministerio de Producción este año, pero solo llegamos a instancias finales del Concurso StartUp Perú que igual nos dejaron grandes experiencias y muchísimas ganas de seguir con este emprendimiento.

\section{Conflictos de interés}

Declaro no tener conflictos de interés en la publicación de este artículo.

\section{Referencias bibliográficas}

Bertram, J. (2012). Clientes de servicios web seguros en dispositivos moviles. ProcediaComputer Science.

Cabero, J., Barroso, J., \& Obrador , M. (2016). Realidad Aumentada aplicada a la enseñanza de la medicina. Educación Médica.

Carbajal Solis, V. (2004). Determinación de la frontera dialectal del quechua ayacuchano y cusqueño en el departamente de Apurimac. Lima, Perú.

Del Moral, M., Villalustre, L., \& Neira, M. (2013). Oportunidades de las TIC para la innovacion educativa en las escuelas rurales de Asturias. Aula Abierta.

Rev. Yachay volumen (7) Número (1), enero-diciembre 2018 
Hintz, D., \& Hintz, D. (2014). La categoría probatoria del conocimiento mutuo en quechua. Lingua.

Merino, C., Pino, S., \& Meyer, E. (2014). Realidad Aumentada para el diseño de secuencias de enseñanza-aprendizaje en química. Educación Química.

Rodríguez H, Pirul J, Robles J, Pérez L, Vásquez E. (2017). Análisis de los estilos de aprendizaje en alumnos de Medicina de la Universidad de Chile. Educación Médica

Santiago Rojano Ramos, M. d. (2016). Desarrollo de tecnologías de la información y la comunicacion para reforzar los procesos de enseñanza y aprendizaje en ciencias en el grado de maestro en educación infantil de la Universidad de Málaga. Educación Química.

Silva, A., \& Martínez, D. (2017). Influencia del Smartphone en los procesos de aprendizaje y enseñanza. Suma de Negocios.

Zaragoza, E., Orozco, L., Nuñez , M., \& Macias, J. (2015). Estrategias didácticas en la enseñanza- aprendizaje: lúdica en el estudio de la nomenclatura química orgánica en alumnos de la Escuela Preparatoria Regional de Atotonilco. Educación Química.
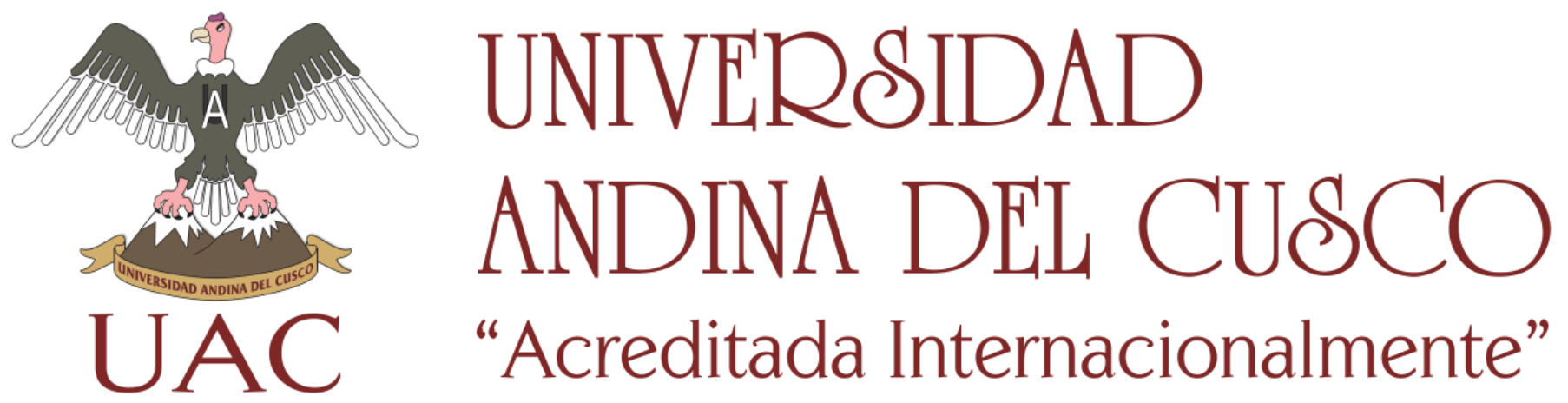

Rev. Yachay volumen (7) Número (1), enero-diciembre 2018 